\title{
Correction: Aberrant ATRX protein expression is associated with poor overall survival in NF1-MPNST
}

\author{
Hsiang-Chih Lu', ${ }^{1,}$, Vanessa Eulo ${ }^{2, *}$, Anthony J. Apicelli, ${ }^{3,4}$, Melike Pekmezci ${ }^{5}, \mathrm{Yu} \mathrm{Tao}^{6}$, \\ Jingqin Luo ${ }^{6}$, Angela C. Hirbe ${ }^{7,4}$ and Sonika Dahiya ${ }^{1,4}$ \\ ${ }^{1}$ Division of Neuropathology, Department of Pathology and Immunology, Washington University School of Medicine, St. \\ Louis, MO, USA \\ ${ }^{2}$ Department of Medicine, Washington University School of Medicine, St. Louis, MO, USA \\ ${ }^{3}$ Department of Radiation Oncology, Washington University School of Medicine, St. Louis, MO, USA \\ ${ }^{4}$ Siteman Cancer Center, Washington University School of Medicine, Saint Louis, MO, USA \\ ${ }^{5}$ Department of Pathology, University of California San Francisco School of Medicine, San Francisco, CA, USA \\ ${ }^{6}$ Siteman Cancer Center Biostatistics Shared Resource, Division of Public Health Sciences, Department of Surgery, Washington \\ University School of Medicine, St. Louis, MO, USA \\ ${ }^{7}$ Division of Medical Oncology, Washington University School of Medicine, St. Louis, MO, USA \\ *Co-first authors
}

Published: November 23, 2021

Copyright: (c) $2021 \mathrm{Lu}$ et al. This is an open access article distributed under the terms of the Creative Commons Attribution License (CC BY 3.0), which permits unrestricted use, distribution, and reproduction in any medium, provided the original author and source are credited.

This article has been corrected: The Supplementary Table 2 has been updated to show the appropriate WUSTL numbers in the first column. The corrected Table 2, produced using the original data, is shown below. The authors declare that these corrections do not change the results or conclusions of this paper.

Original article: Oncotarget. 2018; 9:23018-23028. https://doi.org/10.18632/oncotarget.25195

\section{Supplementary Table 2: Mitotic index}

\begin{tabular}{ccc}
\hline Case & Mitoses & Tumor Type \\
\hline WU-1 & $74 / 10 \mathrm{HPF}$ & MPNST \\
WU-2 & $17 / 10 \mathrm{PF}$ & MPNST \\
WU-3 & $19 / 10 \mathrm{PF}$ & MPNST \\
WU-4 & $28 / 10 \mathrm{HPF}$ & MPNST \\
WU-5 & $78 / 10 \mathrm{HPF}$ & MPNST \\
WU-6 & $4 / 10 \mathrm{HPF}$ & MPNST \\
WU-7 & $6 / 10 \mathrm{HPF}$ & MPNST \\
WU-8 & $5 / 10 \mathrm{HPF}$ & MPNST \\
WU-9 & $81 / 10 \mathrm{HPF}$ & MPNST \\
WU-10 & $11 / 10 \mathrm{HPF}$ & $*$ \\
WU-11 & $25 / 10 \mathrm{HPF}$ & MPNST \\
WU-12 & $3 / 10 \mathrm{HPF}$ & MPNST \\
WU-13 & $8 / 10 \mathrm{HPF}$ & MPNST \\
WU-14 & $25 / 10 \mathrm{HPF}$ & MPNST \\
WU-15 & $32 / 10 \mathrm{HPF}$ & MPNST
\end{tabular}


WU-17

WU-18

WU-19

WU-20

WU-21

WU-22

WU-23

WU-24

WU-25

WU-26

WU-27

WU-28

WU-29

WU-30

WU-31

WU-32

WU-33

WU-34

WU-35

WU-36

WU-37

WU-38

WU-39

WU-40

WU-41

WU-42

WU-43

WU-44

WU-45

WU-46

WU-47

WU-48

WU-49
27/10 HPF

${ }^{* *}$ Metastatic lesion

9/10 HPF

27/10 HPF

6/10 HPF

8/10 HPF

6/10 HPF

4/10 HPF

$3 / 10 \mathrm{HPF}$

23/10 HPF

15/10 HPF

4/10 HPF

20/10 HPF

21/10 HPF

69/10 HPF

9/10HPF

7/10 HPF

55/10 HPF

${ }^{* *}$ Metastatic lesion

23/10 HPF

20/10 HPF

8/10 HPF

14/10 HPF

62/10 HPF

$>1 / 50 \mathrm{HPF}$

$>1 / 50 \mathrm{HPF}$

$>1 / 50 \mathrm{HPF}$

$>1 / 50 \mathrm{HPF}$

$>1 / 50 \mathrm{HPF}$

$>1 / 50 \mathrm{HPF}$

0 (small biopsy)

0 (small biopsy)

0 (small biopsy)
MPNST

MPNST

MPNST

MPNST

MPNST

MPNST

MPNST

MPNST

MPNST

MPNST

MPNST

MPNST

MPNST

MPNST

MPNST

MPNST

MPNST

MPNST

MPNST

MPNST

MPNST

MPNST

MPNST

MPNST

Atypical Neurofibroma

Atypical Neurofibroma

Atypical Neurofibroma

Atypical Neurofibroma

Atypical Neurofibroma

Atypical Neurofibroma

Atypical Neurofibroma

Atypical Neurofibroma

Atypical Neurofibroma

*Brisk mitotic activity per pathology report. All slides not available for current review. ${ }^{* *}$ Mitotic count was not performed on metastatic lesions. 